\title{
Nigella sativa L seed's Extract Modulates Liver Regeneration by Affecting Endogenous Stem Cells in Liver Fibrosis Model of Rat
}

\author{
$\underline{\text { Safithri Fathiyah }}^{1^{*}}$, Putra Suhartono Taat ${ }^{2}$, Ferdiansyah $^{3}$ \\ ${ }^{1}$ Doctorate Student, Postgraduate Programme, Medical Faculty, Airlangga University, \\ Surabaya, Indonesia, \\ ${ }^{2}$ Anatomy Pathobiology Department, Medical Faculty, Airlangga University, Surabaya, \\ Indonesia \\ ${ }^{3}$ Orthopedic Department, RSUD dr Soetomo, Surabaya, Indonesia \\ *e-mail : fathiyahsafithri@yahoo.co.id
}

\begin{abstract}
Chronic liver disease commonly result in liver fibrosis and eventually liver cirrhosis. It has been demonstrated that patients with liver cirrhosis whom consumed $N$. sativa seed'sexperienced an improvement such as increasing of serum albumin level followed by diminished lower extremities oedem and ascites. The increasing of serum albumin level probably caused by repairment of liver parenchym and or function. Our research aim was evaluation of $N$. sativa seed's extracts as hepato regenerative agent againts hepatocellular damage induced by hepatotoxins $\left(\mathrm{CCl}_{4}\right)$. 18 Rats were divided into three groups; normal, liver fibrotic control and liver fibrotic treated with $N$. sativa groups, 6 rats in each. Liver fibrosis induced by $\mathrm{CCl}_{4}$ intraperitoneal injection @ $1 \mathrm{~mL} / \mathrm{kgBB}$ for 8 weeks, three times in week. After $\mathrm{CCl}_{4}$ induction, administrated ethanol extract of $N$. sativa seeds by daily intragastric gayage for 4 weeks. At the end of the study, the animal sacrificed, blood and tissue sample were obtained. The level of albumin serum was examinated by spectrophotometry, the mesenchymal stem cell marker CD90, and hepatoblast marker HNF$4 \alpha$ expressions were examinated with immunohistochemistry. It was found that $N$. sativa's extracts significantly revised on albumin serum level and improved the liver tissue through the increasing of CD90 and HNF-4 $\alpha$ expression. From this study, it concluded that Nigella sativa seed's extract exhibited a promising hepatoregenerative effect in the management of liver fibrosis.
\end{abstract}

Keywords: Nigella sativa, Liver fibrosis, CD90 expression, HNF-4a expression, Albumin serum level

\section{INTRODUCTION}

Chronic liver diseases tend to end up as liver fibrosis and liver cirrhosis. Previous studies have shown that elevation of albumin serum as well as reduction of limb oedema and ascites in a patient with liver cirrhosis are associated with Nigella sativa (N.sativa) consumption [1]. The elevation of albumin serum level is potentially resulted from liver regeneration process, which defined as liver parenchyma refinement or hepatocyte function improvement. The mesenchymal stem cells have a substantial role in hepatocytes regeneration. The endogenous stem cells are quiescent and can be activated into hepatoblasts depends on the influence of the microenvironment of surrounding stem cell (niche)[2]. 
$N$ sativa's seeds that bear multi compounds are expected to be able to affect the stem cell niche and modulate liver regeneration in liver fibrosis. This study aims to prove that N.sativa seeds extract can bring a hepato-regenerative effect that can be seen as elevated serum albumin level as a result of the activation of mesenchymal stem cell activation in rat model of liver fibrosis that being induced by carbon tetrachloride (CCl4). CD90 is one of mesenchymal stem cell marker, and HNF- $4 \alpha$ is the marker of hepatoblast.

\section{METHODS}

Twenty one white male Wistar rats, with 150-200 g of body weight, at 2-3 months old were included in this study. All rats were kept in a standardized laboratory cage. Randomisation was done following the planned scheme. Rats were classified into one out of three groups; group A (normal rats), B ( $\mathrm{CCl} 4$ only) and $\mathrm{C}(\mathrm{CCl} 4+N . s a t i v a)$; each group consist of 7 rats. Group B and C received 10\% $1 \mathrm{~mL} / \mathrm{kgBW}$ of CCl4 injection (Merck; 153,82 $\mathrm{g} / \mathrm{mol}$ ) for 8 weeks, 3x/week. Group C received $2.4 \mathrm{~g} / \mathrm{kgBW}$ oral $N$.sativa extract for four weeks, following eight weeks $\mathrm{CCl} 4$ induction. N.sativa seeds were obtained from Ad-Dawa's Research CV Rizki Abadi Tuban and extracted using ethanol following Andaloussi's method (Andaloussi, 2011). By the end of this study, rats were anaesthetized using $100 \mathrm{mg} / \mathrm{kgBW}$ of Ketamine $\mathrm{HCl}$, intramuscular. Soon afterwards, we drew the blood samples from intracardiac and liver blood. The blood samples were centrifuged to separate the serum. The blood serum was taken, and the albumin level was counted using spectrophotometry. The liver organ samples were fixated in $10 \%$ formalin buffer and processed to become paraffin block. The paraffin blocks were then cutted into $4 \mu \mathrm{m}$-thick slices using rotary microtome and then put onto an object glass. Next, the slices were stained using Immunhistochemistry staining of monoclonal anti-CD90 to measure the CD90 expression and monoclonal antiHNF-4 $\alpha$ to measure the HNF-4 $\alpha$ expression. The observation of CD90 and HNF-4 $\alpha$ was done using light microscope by 1000x magnification. Data was displayed as mean \pm SD and analysed using ANOVA followed by LSD to evaluate the difference between each group. We also used linear regression analysis to perceive the effect of N.sativa extract towards CD90 and HNF-4 $\alpha$ expression and albumin serum level.

\section{RESULTS}

The mean expression of CD-90, HNF-4 $\alpha$, and serum albumin level from all groups are explained in Table 1. CCl4 induction significantly reduces the expression of CD90 $(\mathrm{p}<0.05)$ and serum albumin level $(\mathrm{p}<0.05)$, and increase the expression of HNF-4 $\alpha(\mathrm{p}<0.05)$. N.sativa supplementation also significantly increase the expression of CD90 $(\mathrm{p}<0.05)$ and HNF-4 $\alpha(\mathrm{p}<0.05)$, but not albumin serum level $(\mathrm{p}=0.106)$. The N.sativa supplementation affects the expression of CD90, HNF-4 $\alpha$ and serum albumin level by $95.5 \%, 93.4 \%$, and $17.7 \%$, consecutively. The expression of CD90 affect serum albumin level by $88.3 \%$, but the expression of HNF-4 $\alpha$ affect the serum albumin level by $19.1 \%$. 
Table 1. The mean expression of CD90, expression of HNF-4 $\alpha$ and serum albumin level in normal rats (A), rats injected by only $\mathrm{CCl} 4$ (B), and rats injected by $\mathrm{CCl} 4$ and given N.sativa supplementation (C).

\begin{tabular}{lccc}
\hline & A & B & C \\
\hline CD90 expression & $3,3 \pm 0,8$ & $2,0 \pm 0,6$ & $7,8 \pm 0,8$ \\
HNF-4aexpression & $2,7 \pm 0,8$ & $6,0 \pm 0,9$ & $13,3 \pm 1,2$ \\
Serum albumin level & $2,1 \pm 0,1$ & $1,5 \pm 0,4$ & $1,8 \pm 0,3$ \\
\hline
\end{tabular}
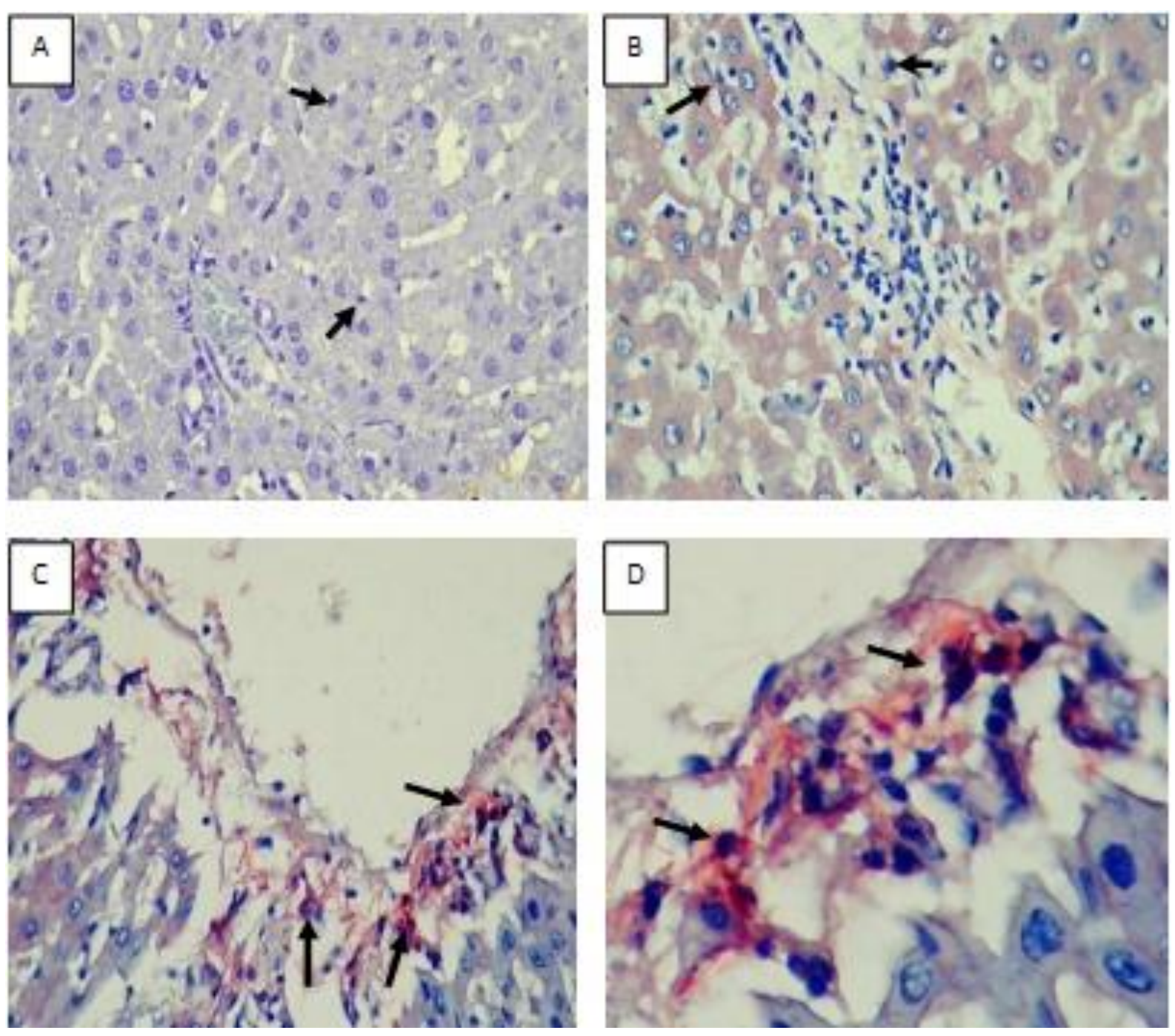

Figure 1. The expression of CD90 in normal rats (A), rats injected by only $\mathrm{CCl}_{4}(\mathrm{~B})$, and rats injected by $\mathrm{CCl}_{4}$ and given N.sativa supplementation (C) at 400x magnification N.sativa, and the expression of CD90 at 1000x magnification N.sativa. The black arrows show positive expression of CD90, marked as a red-stained cell membrane. 

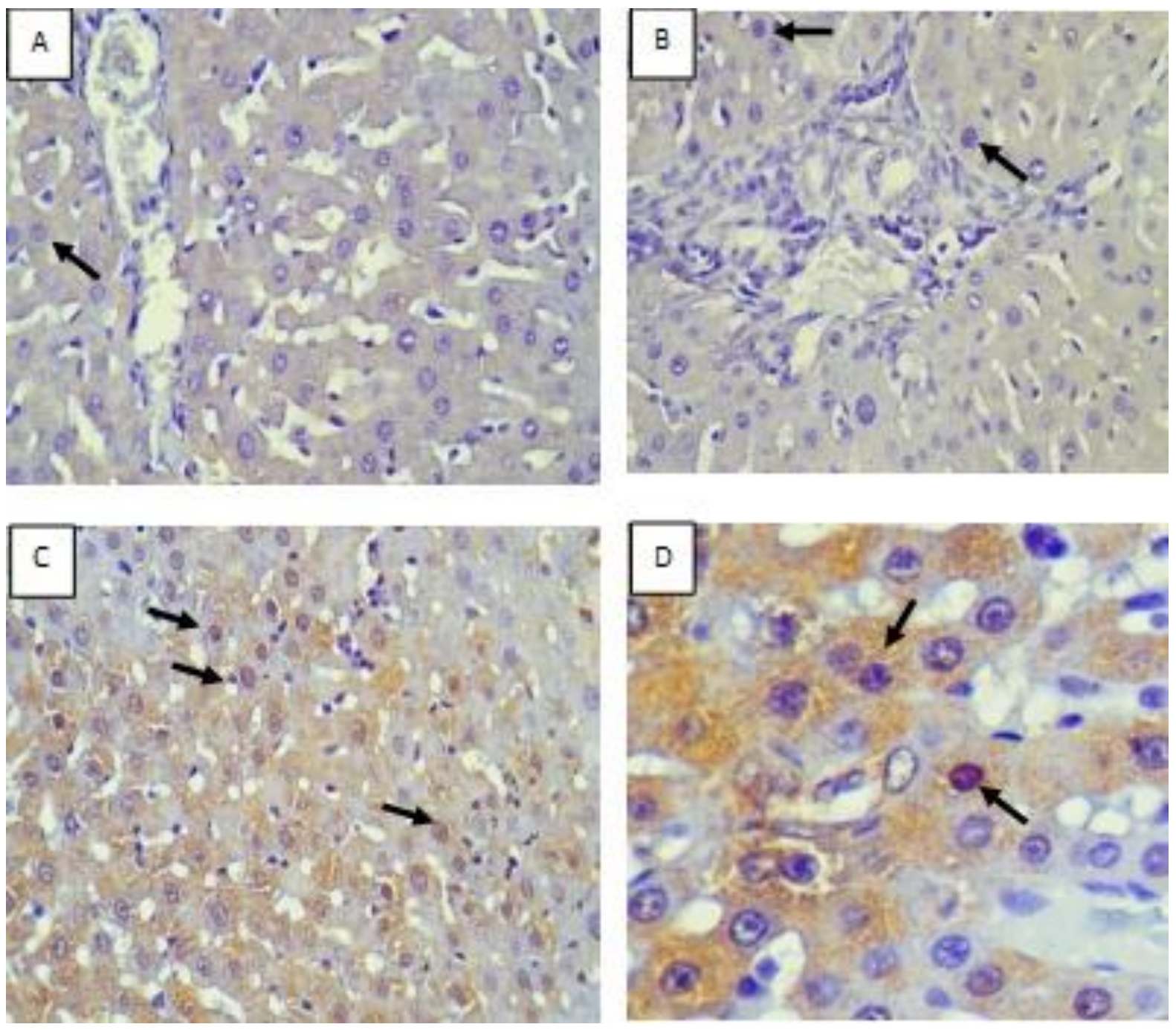

Figure 2. The expression of HNF-4ain normal rats (A),rats injected by only CCl4 (B), and rats injected by $\mathrm{CCl} 4$ and given N.sativa supplementation (C) at 400x magnificatioN.sativa, and the expression of HNF-4 $\alpha$ at 1000x magnification N.sativa. The black arrows show positive expression of HNF- $4 \alpha$, marked as brown-stained nucleus in hepatocytes.

\section{DISCUSSION}

N.sativa extract contains active ingredients, such as thymoquinone, oleic acid and linoleic acid[3]. N. sativa extract supplementation increases the expression of CD90 in the liver and gave $95.5 \%$ influence, showing that N.sativa extract has a potential to increase the MSC homing and migration from bone marrow to liver and/or increase the MSC proliferation in liver. MSC is important in liver regeneration. MSC can be differentiated into hepatocytelike cells both in vitro and in vivo[4]. We predict that these effects are resulted from the linoleic acid and thymoquinone compounds in N.sativa extract. Previous invitro study by Smith, 2012 showed that linoleic acid exposure on human MSC for seven consecutive days in vitro was associated with increased migration ability of stem cell in $2 \%$ fetal bovine serum (FBS) medium[5]. Thymoquine is G-protein couple receptor agonist that can activates p38- 
mitogen-protein kinase (p38/MAPK) pathway[6]. Activation of MAPK pathway can activate Wnt/ $\beta$-catenin, and then activate cyclin D2 that responsible on mesenchymal stem cell proliferation[7]. Activation of MAPK can activate transcription regulator protein, such as cmyc, HGF, cyclin D that can induct the quiscent endogenous mesenchymal stem cell activation[8].

N.sativa extract supplementation also increases the expression of HNF-4 $\alpha$ by $93.3 \%$ and the expression of CD90 influence the expression of HNF-4 $\alpha$ by $88.3 \%$, showing that N.sativa has a potential to increase the number of hepatoblasts by upgrading the differentiation ability from MSC into hepatoblasts. Thymoquinone (TQ) as the primary bioactive compounds of N.sativa are suspected as the main actor of this differentiation process. In vitro study by Wirries in 2013 showed that TQ plays a role in progenitor cell differentiation in osteogenesis [9].

N.sativa extract also increase the serum albumin level, but the elevation level is not significant and only give small influence by $17.7 \%$. The expression of HNF-4 $\alpha$ affects the level of serum albumin level by $19.1 \%$. The elevation of serum albumin level reflects the improvement in liver function, which can be resulted from the damaged cells replacement by the new healthy cells. These new hepatocytes are potentially arised from hepatoblasts maturation. Hepatoblasts do not have a protein synthesis function. In addition, albumin has a long duration of action, for 3-4 weeks. The non-significant elevation may be caused by the short duration of N.sativa exposure, which only lasts for four weeks, while the duration for hepatoblasts maturation into mature hepatocytes need longer duration. Therefore, N.sativa supplementation for four weeks, though showing a correlation with the elevation of albumin level, can only give a non-significant result.

\section{REFERENCES}

[1] Barakat EMF, Wakeel LME, Hagag RS, 2013, Effects of Nigella sativa on outcome of Hepatitis C in Egypt, World Journals of Gastroenterology 28; 19(16): 2529-2536

[2] Ma S, Xie N, Li W, Yuan B, Shi Y, dan Wang Y, 2014, Immunobiology of mesenchymal stem cells, Cell Death and Differentiation 21; 216-225

[3] Ahmed SK, Mohammed SA, Khalaf G, Fikry H, 2014, Role of Bone Marrow Mesenchymal Stem Cells in the Treatment of CCL4 Induced Liver Fibrosis in Albino Rats: A Histological and Immunohistochemical Study, International Journal of Stem Cells Volume. 7, No. 2, pp.87-97

[4] Eom YW, Shim KY, and Baik SK, 2015, Mesenchymal stem cell therapy for liver fibrosis, Korean Journal Internal Medicine 30:580-589

[5] Smith AN, Muffley LA, Bell AN, Numhom S, dan Hocking AM, 2012, Unsaturated fatty acids induce mesenchymal stem cells to increase secretion of angiogenic mediators, Journal Cell Physiology ; 227(9): 3225-3233 
[6] Finlay TM, Abdulkhalek S, Gilmour A, 2010. Thymoquinone-induced Neu4 sialidase activates NFkappaB in macrophage cells and proinflammatory cytokines in vivo. Glycoconjugation Journal ;27(6):583-600

[7] Stewart AF, Hussain MA, Ocana AG, Vasavada RC, Bhusnan A, Bernal-Mizrachi E Kulkarni RN, Mizrachi EB, 2015, Human $\beta$-cell proliferation and intracellular signaling: Part 3, Diabetes 64:1872-1885

[8] Roux PP and Blenis J, 2004, ERK and p38 MAPK-Activated Protein Kinases: a Family of Protein Kinases with Diverse Biological Functions, Microbiology And Molecular Biology Reviews, Vol. 68, No. 2, p. 320-344

[9] Wirries A, Schubert A, Zimmermann R, Jabari S, Ruchholtz S, El-Najjar N, 2013, Thymoquinone accelerates osteoblast differentiation and activates bone morphogenetic protein-2 and ERK pathway, International Immunopharmacology 15:381-386 